\title{
Erythropoietin and Breast Cancer Progression: An in vitro Study
}

\author{
J. Obayemi**, A. Calabro*, C. Queenan*, D. Becker*, D. Leonardi** \\ * Bergen County Academies, Nano-Structural Imaging Lab, 200 Hackensack Avenue, Hackensack, \\ NJ 07601 \\ ** Bergen County Academies, Biotechnology Lab, 200 Hackensack Avenue, Hackensack, NJ 07601
}

Erythropoietin (EPO) is a hormone produced in the kidneys that regulates the production of red blood cells. When a patient is undergoing chemotherapy, anemia often ensues and recombinant EPO is used as treatment. In 2007, the FDA imposed a Black Box Warning on EPO for chemotherapyinduced anemia due to the increased incidence of tumor progression associated with its use in patients with head, neck and breast cancers. Recently, statins, commonly used for the reduction of cholesterol, have been suggested to have antitumor effects and may be a potential treatment option for this tumor progression exacerbated by EPO therapy [1]. The goal of this in vitro study was to evaluate the relationship between EPO and breast cancer and to determine if a common statin, Lovastatin, would have any effect during EPO administration.

MDA-MB-231 and MCF-7 breast cancer cells were assayed to determine the effects of exogenous EPO in both normoxia $\left(21 \% \mathrm{O}_{2}\right)$ and hypoxia $\left(3 \% \mathrm{O}_{2}\right)$; the latter used to simulate the tumor environment. Results demonstrated that $1 \mathrm{U} / \mathrm{mL}$ EPO increased proliferation $(\mathrm{p}<0.05)$ and resulted in resistance to the LD50 of cisplatin ( $\mathrm{p}<0.05)$, a chemotherapeutic agent, in both normoxia and hypoxia. Hypoxia significantly increased the secretion of endogenous EPO $(p<0.05)$. Administration of Lovastatin $(10-100 \mu \mathrm{M})$ demonstrated a statistically significant down regulation of the EPO receptor and induced apoptosis $(\mathrm{p}<0.05)$ in both hypoxia and normoxia, supporting its use to reduce the deleterious effects of EPO during treatment for chemotherapy-induced anemia. To increase the membrane permeability of Lovastatin, two types of carrier molecules, 40nm-gold nanoparticles coated with cyclodextrin and liposomal capsules, were employed. Delivery of Lovastatin via gold nanoparticles was determined to enhance cancer cell proliferation, most likely due to a low-dose effect; however, liposomes were able to significantly decrease proliferation $(\mathrm{p}<0.05)$ more effectively than Lovastatin alone.

Transmission electron microscopy (TEM) was used to confirm incorporation of the nanoparticles into the cancer cells. Cells treated with the gold nanoparticle carriers were fixed in $4 \%$ glutaraldehyde $/ 2 \%$ formaldehyde in $0.2 \mathrm{M}$ sodium cacodylate buffer, $\mathrm{pH} 7.4$, scraped from the wells with Teflon ${ }^{\circledR}$ and gently spun down to form a pellet. The pellet was post-fixed in $2 \%$ osmium tetroxide, dehydrated in a graded series of acetone, infiltrated and cured in epoxy resin. 100nm sections were collected onto 200 mesh copper grids and post-stained with $4 \%$ uranyl acetate and $0.5 \%$ lead citrate. Liposomal carriers were negatively stained with $2 \%$ uranyl acetate prior to imaging.

TEM images of cells treated with the gold nanoparticle Lovastatin carriers confirmed that the carriers were able to enter the cells (Figure 1). Furthermore, images showed that the carriers tended to aggregate, often inside a membrane-bound vesicle. TEM analysis of the liposomes (Figure 2) showed the structure of the liposomal drug carriers. Based on the results of this study, the 
proliferation of cancer cells caused by EPO can be mediated by combinatorial treatment with Lovastatin, which was shown to be most effectively delivered into cells by liposomal carriers.

References

[1] G. Acs, et al., Cancer Letters. 170 (2004) 243-251.

[2] The authors would like to acknowledge the administration of the Bergen County Technical Schools for their continued support of the research program.
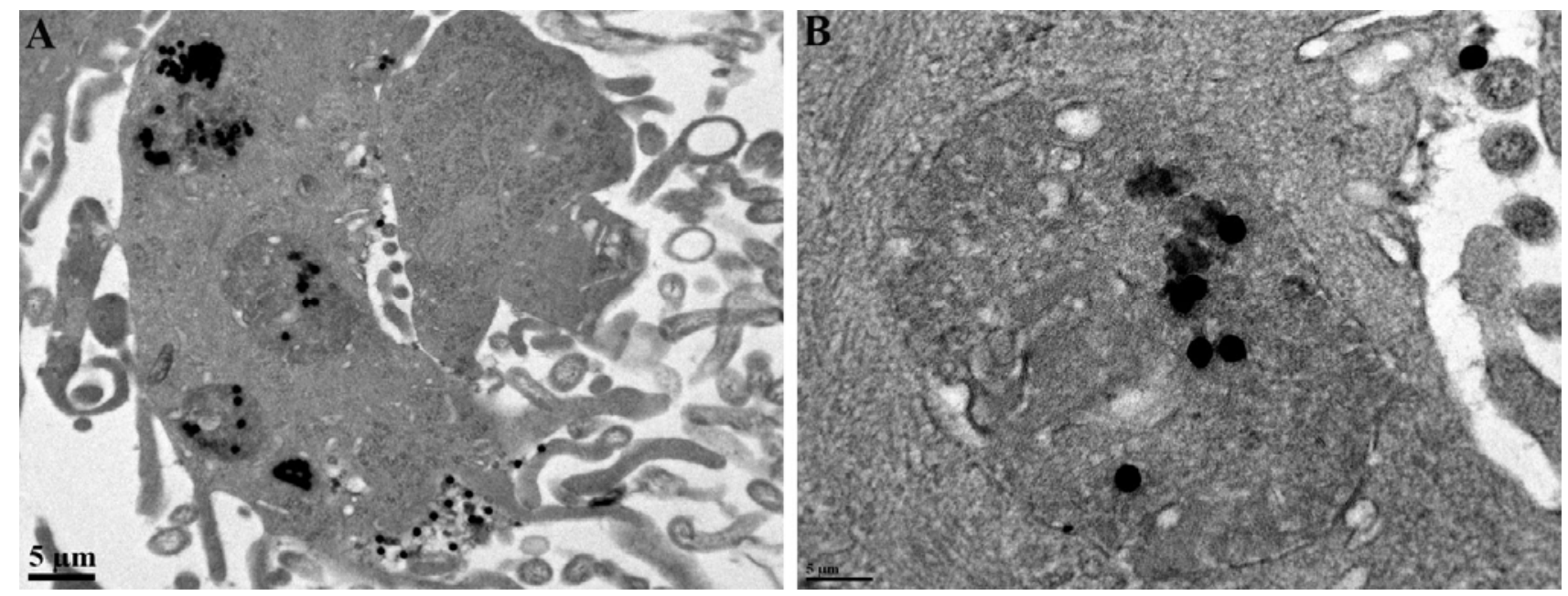

Figure 1: TEM micrographs of gold nanoparticle carriers inside breast cancer cells. (A) Aggregates of nanoparticle carriers inside vesicles. (B) Vesicle containing carrier particles.

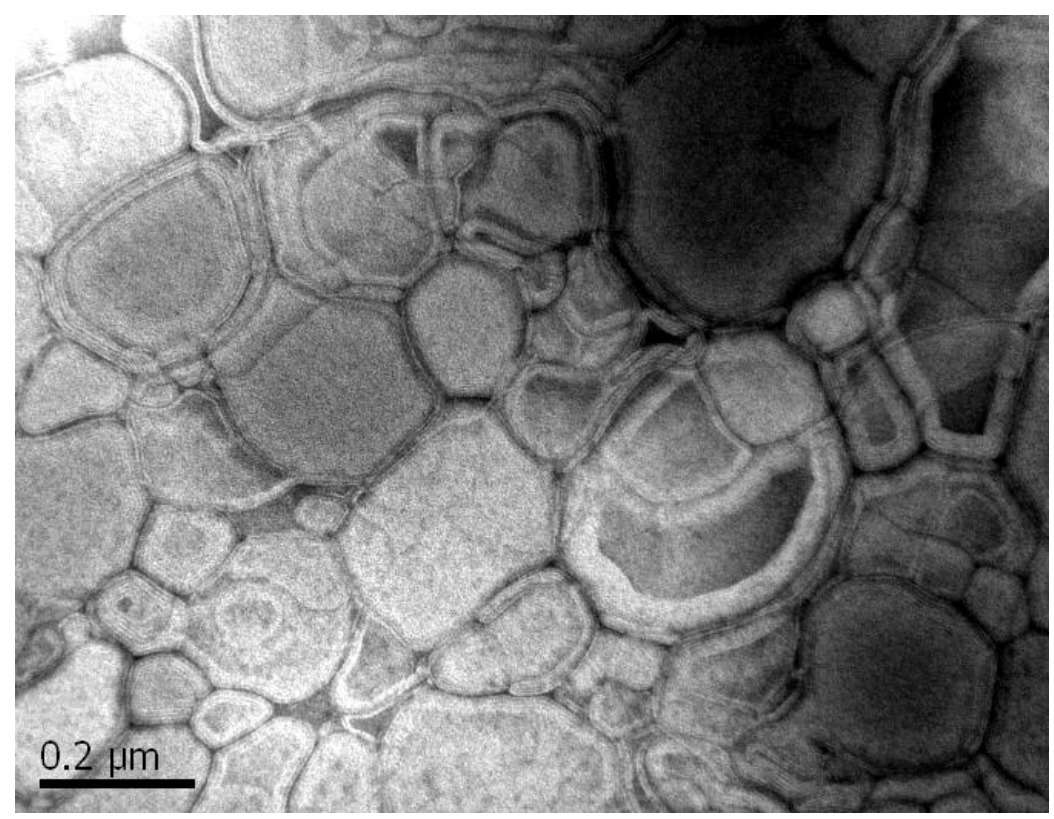

Figure 2: TEM micrograph of liposomal drug carriers. Phospholipid bilayers that comprise the membranes of the liposomes are visible. 